\title{
Improved retinex image enhancement algorithm based on bilateral
} filtering

\author{
Ya'nan Yang ${ }^{1,2, a}$, Zhaohui Jiang ${ }^{1, b,{ }^{*}, \text { Chunhe Yang }}{ }^{1, c}$, Zhiqiang Xia ${ }^{2, d}$, Feng \\ $\mathrm{Liu}^{2, \mathrm{e}}$
}

${ }^{1}$ College of Information \& Computer, Anhui Agricultural University, Hefei, Anhui, 230036, China

${ }^{2}$ College of Communication \& Information Engineering, Nanjing University of Posts and Telecommunications, Nanjing, Jiangsu, 210003, China

ayangyanan919@163.com, bjiangzh@ahau.edu.cn, '951265920@qq.com,

d1244316501@qq.com, ${ }^{\text {liuf@nupt.edu.cn }}$

Corresponding Author: Zhaohui Jiang

Keywords: image enhancement; halo; color distortion; Retinex; bilateral filtering.

\begin{abstract}
In view of such problems as halo and color distortion for the traditional Retinex enhancement algorithm, an improved Retinex image enhancement algorithm based on bilateral filtering was proposed. In the HSI color space, firstly, the gamma correction was utilized to adjust the brightness of the image. And then, the image was decomposed into the illuminant image and the reflected image using the bilateral filter. Afterwards, the dynamic range of the illuminant image was compressed and the local contrast of the reflected image was enhanced. Finally, the saturation S was adjusted adaptively according to the correlation coefficient between the saturation $\mathrm{S}$ and the brightness I. The experimental results indicated that, the improved algorithm could not only suppress the halo and color distortion effectively, but maintain the color saturation and image authenticity, when was applied to color image. Thus, it was very conducive to the practical application of computer vision technology.
\end{abstract}

\section{Introduction}

The uneven illumination, particularly high dynamic lighting environment, causes many problems. For example, the images acquired by digital cameras, camcorders, or other terminals tend to be too bright or dark in certain areas, the target is difficult to be identified and some other issues.

To solve the problems for the quality of image acquisition caused by uneven illumination, a series of methods had been put forward successively, including histogram equalization, image enhancement in gradient field, homomorphic filter and the Retinex theory. Among these methods, the Retinex has become a research hotspot recently as it could achieve a good balance in dynamic range compression, edge enhancement and color constancy [1]. There are three classical algorithms based on the Retinex theory, they are SSR (Single Scale Retinex), MSR (Single Scale Retinex) and MSRCR (Multi-Scale Retinex with Color Restoration). However, SSR could only choose one dimension, it was difficult to simultaneously ensure the dynamic range compression and color fidelity $[1,2]$. Although MSR can ensure the dynamic range compression and color fidelity, it could not achieve the high color fidelity, as it simply weighted the different scales linearly [3]. While MSRCR could achieve color fidelity much further, it was not conducive to real-time applications, because a lot of parameters needed to be set [4]. Furthermore, the above three algorithms may produce halo and color distortion in high contrast regions [5], since the illuminant image was obtained by Gaussian low-filter.

In order to overcome such problems as halo and color distortion for the traditional Retinex, many improved algorithms have been proposed. For example, the halo was reduced through bilateral filter to obtain the illuminant image, but due to being ignorant of the correlations between each channel of the color image, it could not decrease the color distortion [6]. Based on this, some researchers combined the Weber's law converted the image into HSV space, which realized the reduction of 
both halo and color distortion. However, it was not conducive to real-time application, as a lot of parameters needed to be set [7].

To further reduce halo and color distortion, this paper has proposed an improved Retinex image enhancement algorithm based on bilateral filter. And the results demonstrated that it could suppress both the halo and color distortion effectively.

\section{Retinex theory and its typical algorithms}

Retinex (abbreviation of Retina and Cortex) theory is a model been used to explain how the human visual system perceive color and brightness of objects $[8,9]$. It is also the interpretation of color constancy indicating the color of the same objects is constant, though they are under different lighting. Briefly, the basic principle of Retinex theory is that the original image can be divided into illuminant and reflected image, and the enhancement can be achieved through reducing the effects of illuminant image on the reflected. AS shown as in formula (1):

$$
S(x, y)=R(x, y) * L(x, y)
$$

Where $L(x, y)$ represents incident light or illuminant image deciding the dynamic range achieved by image pixels; $R(x, y)$ represents reflective properties or reflected light deciding the interior properties of image; $S(x, y)$ represents the original image. And the visual effects can be improved, mainly by obtaining the reflective properties or reflected image.

\subsection{Single Scale Retinex (SSR)}

The SSR algorithm based on center/surround Retinex approved by Edwin Land is firstly improved and realized by Jobson and his colleagues [10]. And the illuminant image is also obtained by Gaussian low-filter as in formula (2):

$$
R_{S S R}(x, y)=\log S(x, y)-\log (S(x, y) \otimes F(x, y))
$$

Where the symbol $\otimes$ represents convolution operation; $F(x, y)$ is Gaussian surrounding function as in formula (3), (4):

$$
\begin{aligned}
& F(x, y)=\lambda \exp \left[-\frac{\left(x^{2}+y^{2}\right)}{\sigma^{2}}\right] \\
& \iint F(x, y) d x d y=1
\end{aligned}
$$

Where $\lambda$ is a normalization constant; $\sigma$ is the scale of surrounding function, and the smaller $\sigma$ is, the more contrast is enhanced, but the more distinct the halo phenomenon becomes. Conversely, the larger $\sigma$ is, although the less contrast is enhanced, the more effectively the halo is suppressed. Large numbers of experiments indicate that, if $\sigma$ is between 80 and 100, the balance can be maintained.

\subsection{Multi-scale Retinex （MSR）}

MSR with the characteristics of high, medium and low dimensions is a summary of SSR. The specific expression is described as follows:

$$
R_{M S R, i}=\sum_{k=1}^{K} W_{k} R_{S S R, i}
$$

Where $W_{k}$ represents the weights associated with Gaussian function; $k$ represents the number of Gaussian function or surrounding scale. And when $k$ equals to 1 , the MSR will turn into SSR. For color images, $R_{S S R, i}$ represents the SSR result of scale $k$ and channel $i$.

\subsection{Multi-Scale Retinex with Color Restoration （MSRCR）}

The MSRCR algorithm uses a set of color recovery factors, and then multiplies by the result of MSR as in formula (6), (7):

$$
\begin{gathered}
R_{M S R C R, i}(x, y)=G_{i}(x, y) * R_{M S R, i}(x, y) \\
G_{i}(x, y)=\log \left(a * S_{i}(x, y)\right)-\log \left[\sum_{i=1}^{N} S_{i}(x, y)\right]
\end{gathered}
$$


Where $G_{i}(x, y)$ is the color recovery factor. Lots of experiments show that, though the color recovery factor can increase the saturation, there is still some color distortion.

\section{Improved Retinex algorithm based on bilateral filter}

3.1 Improvement thought

1) Conversion to HSI color space

Traditional Retinex took the same measures on three channels of color image, which may lead to the color distortion [11]. To avoid mutual interference between respective channels, all operations of our method were completed in the HSI space.

2) Enhancement of channel I

As the brightness of the original image was low, the contrast was not high. So, the improved gamma correction [6] was utilized to enhance contrast and the details of original image. And the improved gamma correction is described as follows:
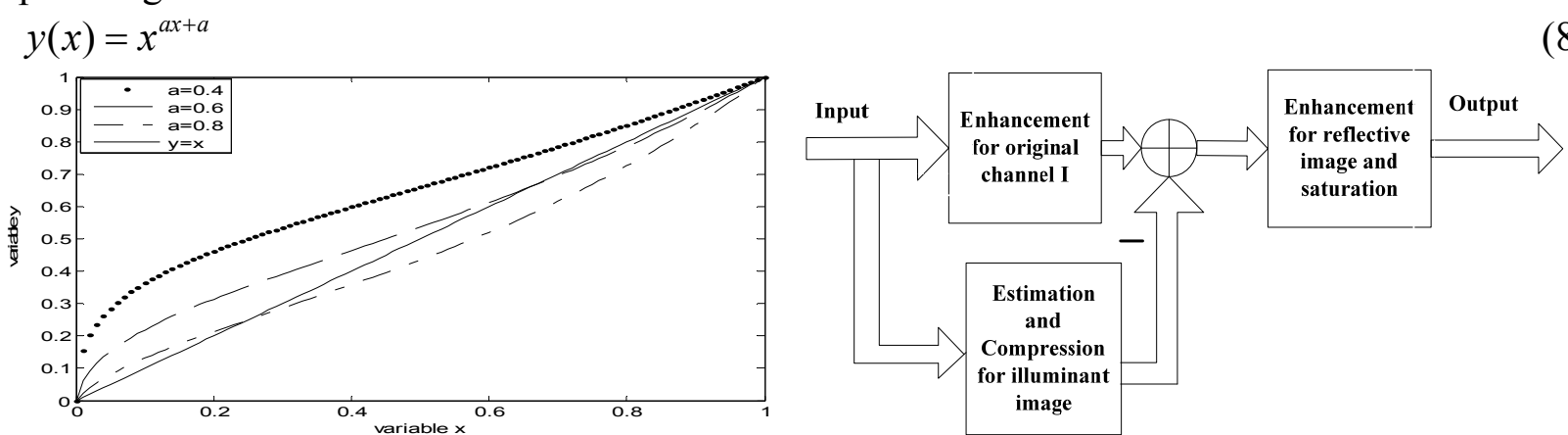

Figure 1.The curve of improved gamma correction Figure 2. The flowchart of improved algorithm

Where ${ }^{x}$ original pixel is value; $y(x)$ is the output value; $a$ is the control parameter, between 0 and 1 . And different values of $a$ represents different correction function. The brightness of image can be mapped through adjusting parameter $a$, as in figure 1: the smaller $a$ is, the more effective for some images whose overall brightness is small. Conversely, the larger $a$ is, the more effective for some images whose overall brightness is larger.

3) Estimation of illuminant image by bilateral filter

As the Gaussian filter characterized by isotropy and only considering the position of the surrounding pixels which may result in halo. So, this paper adopted the anisotropic bilateral filter to estimate the illuminant image more accurately, which considered both the location and the value of the surrounding pixels [12].

4) Compression of illuminant image

For the purpose of meeting hypothesis proposed by the Retinex, which the light changes were gradual. The improved gamma correction was used to compress the dynamic range of illuminant image, and reduce the rapid changes in actual lighting.

5) Enhancement of reflected image and saturation $S$

In order to further highlight the image details and achieve better visual effects, the local contrast enhancement based on local statistical method was adopted on the reflected image [13]. As the image enhancement based on HSI space only enhanced the brightness, the color sense would be inconsistent with the original [14]. Thus, we adjusted the saturation $\mathrm{S}$ adaptively according to the correlation coefficient between the saturation $\mathrm{S}$ and the brightness I.

3.2 Implementation steps

1) Compression of the dynamic range

The gamma correction was used to enhance the channel I as in formula (9):

$v^{\prime}(x, y)=y(v(x, y))$

Where $v^{\prime}(x, y), v(x, y)$ respectively represents the value of channel I before and after enhancement.

2) Estimation and compression for illuminant image 
a) Estimation for the illuminant image

The estimation for illuminant image by bilateral filter is described as follows:

$h(x)=k^{-1}(x) \int_{-\infty}^{\infty} \int_{-\infty}^{\infty} v(x) c(\varepsilon, x) s(v(\varepsilon), v(x)) d \varepsilon$

Where $k(x)=\int_{-\infty}^{\infty} \int_{-\infty}^{\infty} c(\varepsilon, x) s(v(\varepsilon), v(x)) d \varepsilon, c(\varepsilon, x)=e^{-\frac{1}{2}\left(\frac{d(\varepsilon, x)}{\delta_{d}}\right)^{2}}, s(v(\varepsilon), v(x))=e^{-\frac{1}{2}\left(\frac{\delta(v(\varepsilon), v(x))}{\delta_{\gamma}}\right)^{2}}$, and $d(\varepsilon, x)=\|\varepsilon-x\|, \quad c(\varepsilon, x), s(v(\varepsilon), v(x))$ respectively represents the Euclidean distance, distance similarity and brightness similarity between adjacent point $\varepsilon$ and the central point $x ; h(x)$ is the brightness of output image.

b) Compression for the illuminant image

$h^{\prime}(x)=y(h(x))$

3) Decomposition and enhancement of the reflected image

$g(x, y)=v^{\prime}(x, y)-\beta h^{\prime}(x, y)$

$g^{\prime}(x, y)=\bar{g}(x, y)+k \times[g(x, y)-\bar{g}(x, y)]$

Where $g^{\prime}(x, y), g(x, y)$ respectively represents the brightness of output and input; $\bar{g}(x, y)$ represents neighborhood mean of $3 \times 3$ regions; $k$ is the gain coefficient, between 0 and 1 , and the more $k$ is, the more obvious the effect is. The weighting coefficient $\beta$, between 0 and 1 , was added to realize the partial elimination of illuminant image.

4) Adjustment for channel $\mathrm{S}$ to make the color more rich and natural

$s^{\prime}(x, y)=s(x, y)+t\left(v^{\prime}(x, y)-v(x, y)\right) \times \lambda(x, y)$

Where $s(x, y), s^{\prime}(x, y)$ respectively represents the saturation before and after correction. $t$ is a proportional coefficient, between 0 and $1 ; \lambda(x, y)$ is correlation coefficient between saturation $\mathrm{S}$ and brightness I before correction.

The figure 2 gives an overall summary of the proposed method.

\section{Experimental results and discussion}

To validate the performance of the proposed method, we took four images commonly used in high dynamic light environment for experimental objects, and comparing with SSR, MSR and MSRCR from subjective and objective aspects.

4.1 Subjective evaluation

From figure 3(a), 3(b), 3(c) and 3(d), we could see that the overall contrast was poor. And from the corresponding histograms, as the probability of pixels with low gray value was relatively higher than high value ones, thus, the overall gray scale of the image was low.

The images enhanced by SSR, MSR, MSRCR, although the details and contrast was enhanced, there was excessive enhancement and color distortion. However, the color distortion for MSRCR was slightly weakened. And from the corresponding histograms, we could also see that the gray scale distributions were basically the same. As shown in figure 3(h), 3(l), 3(p) and 3(t), the proposed method could not only achieve detail enhancement in dark areas and suppress the excessive enhancement, color distortion, but also maintain the authenticity. And from the corresponding histograms, we could see distribution was more uniform and the contrast was higher.

4.2 Objective evaluation

This paper compared these methods from the clarity, entropy and the running time. And the higher clarity is, the more rich the image details are, the sharper the outline of object edge becomes [15]. The larger entropy represents more information and more obvious details of the texture are. The shorter running time denotes the algorithm is much more real-time. All these three indicators are described as follows:

1) Clarity 


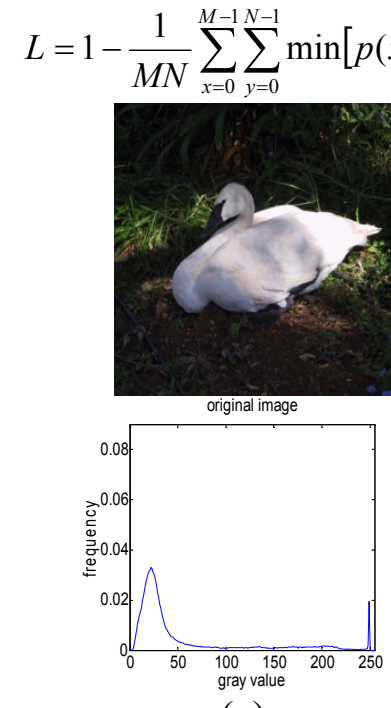

(a)

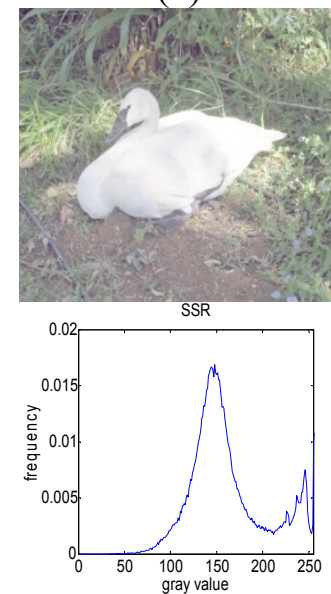

(e)
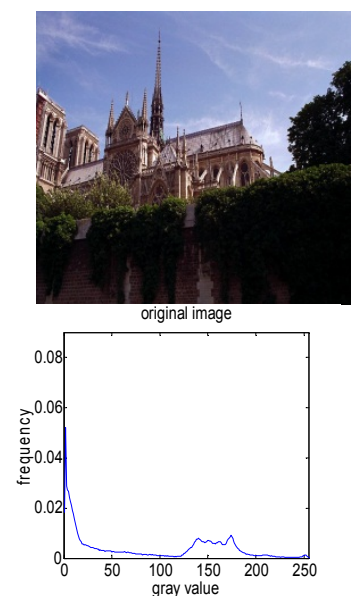

(b)

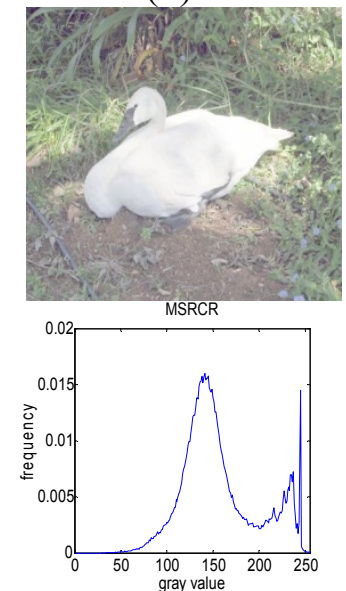

(f)
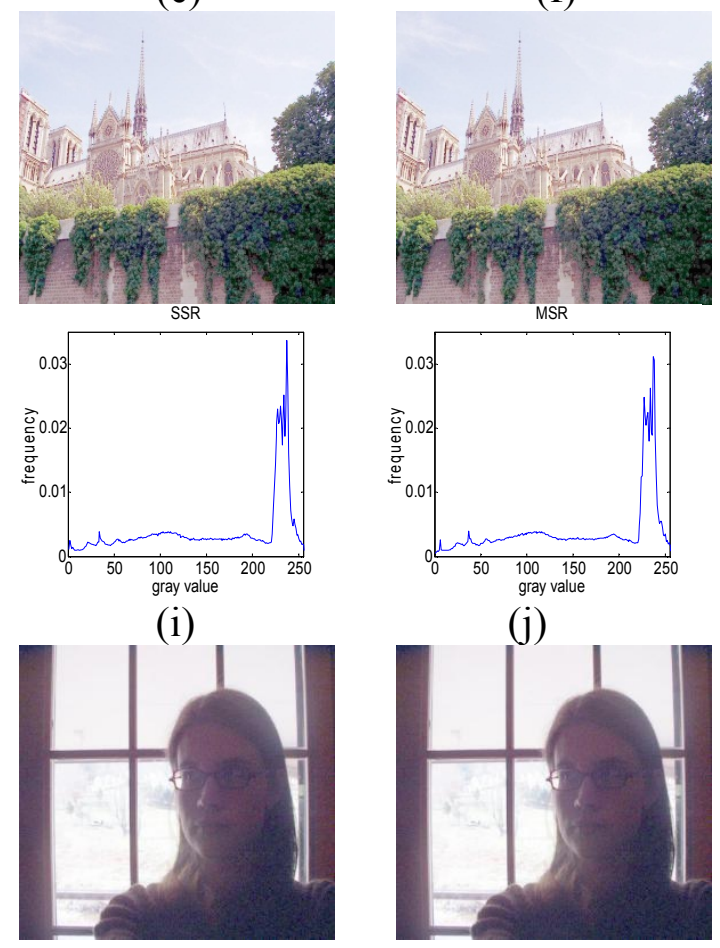
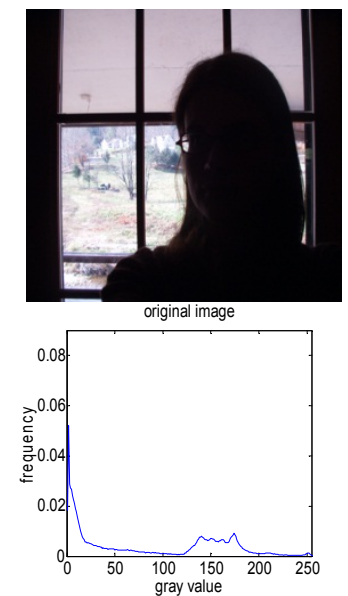

(c)
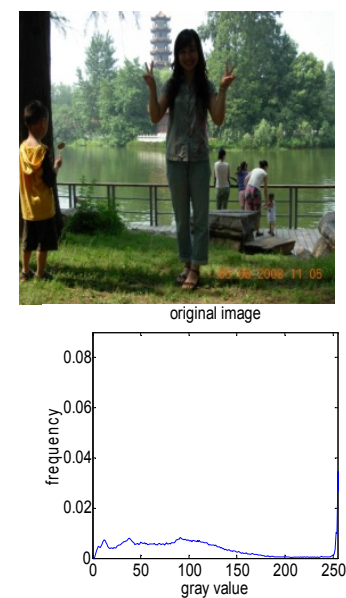

(d)

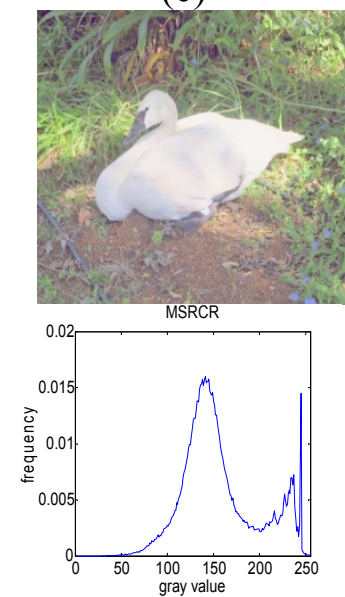

(g)
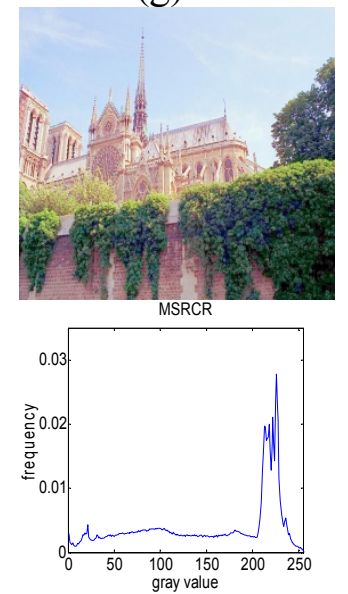

(k)
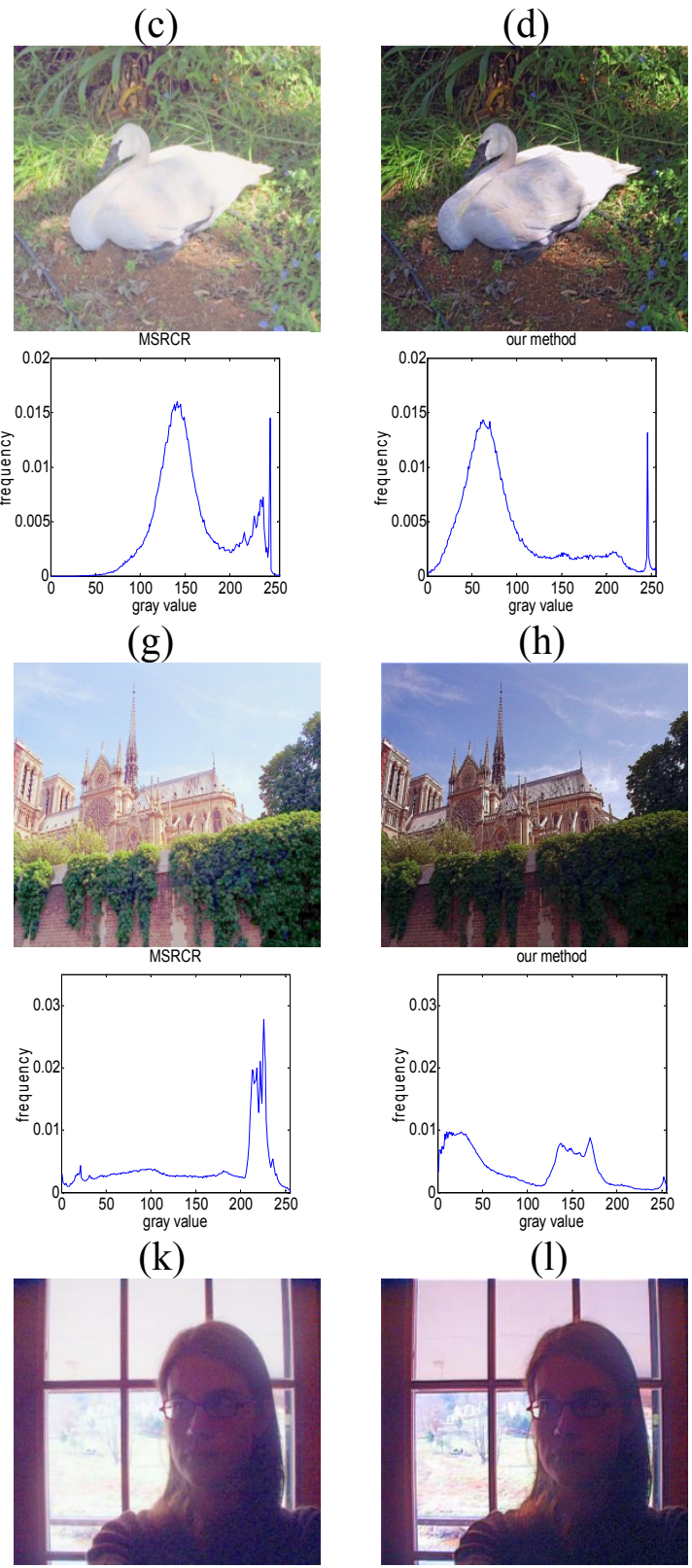

(h)
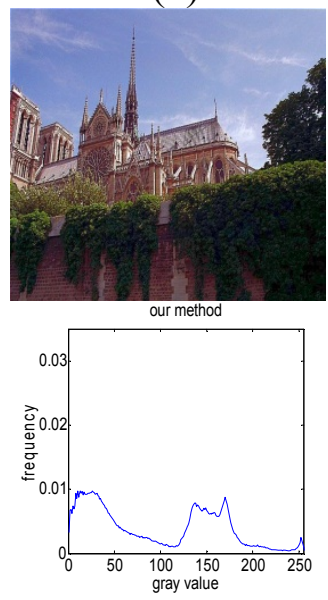

(1)

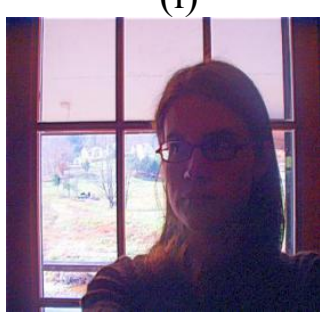




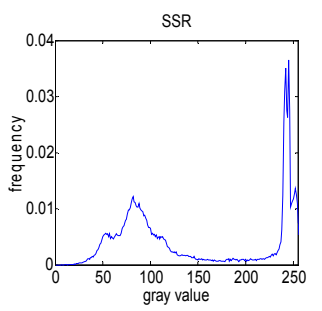

(m)
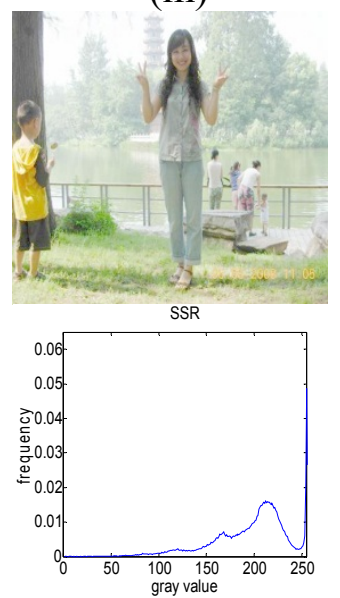

(q)

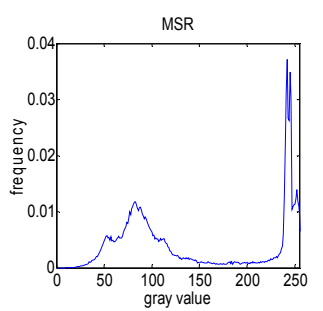

(n)
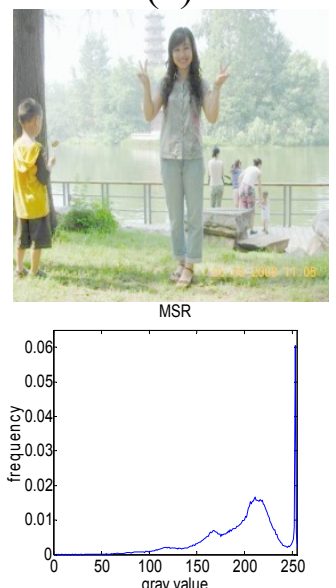

(r)

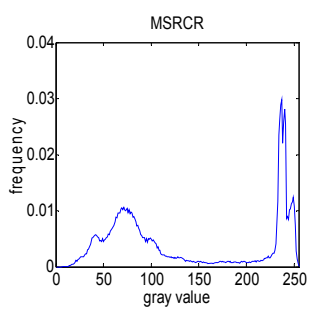

(o)
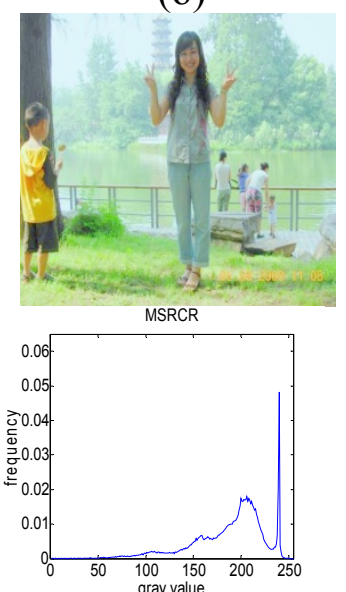

(s)

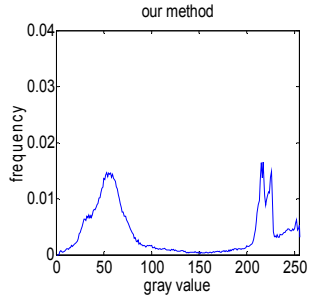

(p)
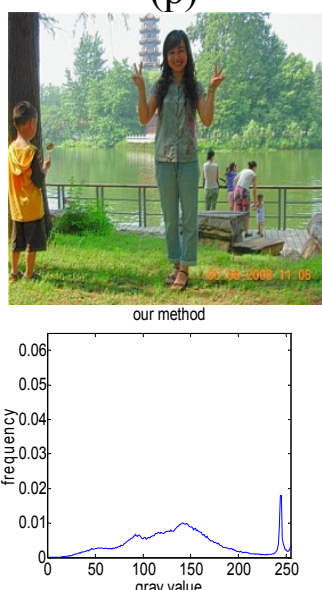

(t)

Figure 3. Experimental results and the corresponding histograms

Where $p(x, y)=\sin \left[\frac{\pi}{2} \times\left(1-f(x, y) / f_{\max }\right)\right] ; f_{\max }$ represents the maximal gray pixel value.

2) Entropy

$$
E=-\sum_{x=1}^{m} \sum_{y=1}^{n} p(x, y) \log p(x, y)
$$

Where $p(x, y)$ represents the gray scale of pixel $(x, y)$.

From table 1, we could see the clarity and information entropy of figure 3(1), 3(b) was improved comparing with the original image. However, the clarity of the two images, and the entropy of figure 3(d) was not improved. Besides, the indicator values had little difference between the three algorithms. For our method, the entropy had different degrees of increase, comparing with the image before and processed by SSR, MSR, and was only slightly lower than MSRCR. In addition, the clarity was much larger than the original image and the three former algorithms. Since the enhancement effect was improved at algorithm complexity expense, the running speed was followed by SSR, MSR, MSRCR and our method from fast to slow.

Table 1 Indicator test results

\begin{tabular}{|c|c|c|c|c|c|c|c|c|c|c|c|c|}
\hline & \multicolumn{3}{|c|}{ 3(a) } & \multicolumn{3}{|c|}{ 3(b) } & \multicolumn{3}{|c|}{$3(\mathrm{c})$} & \multicolumn{3}{|c|}{$3(\mathrm{~d})$} \\
\hline & $\mathrm{C}$ & $\mathrm{E}$ & $\mathrm{T}$ & $\mathrm{C}$ & $\mathrm{E}$ & $\mathrm{T}$ & $\mathrm{C}$ & $\mathrm{E}$ & $\mathrm{T}$ & $\begin{array}{l}\mathrm{C} \\
\end{array}$ & $\mathrm{E}$ & $\mathrm{T}$ \\
\hline Original & 7.87 & 6.56 & --- & 12.12 & 7.34 & --- & 5.99 & 6.39 & --- & 12.41 & 7.46 & --- \\
\hline SSR & 10.11 & 7.06 & 3.75 & 16.98 & 7.58 & 3.81 & 5.42 & 7.10 & 2.82 & 7.18 & 7.38 & 1.59 \\
\hline MSR & 10.25 & 7.08 & 3.91 & 16.94 & 7.58 & 4.01 & 5.41 & 7.10 & 2.93 & 7.66 & 7.15 & 1.70 \\
\hline MSRCR & 10.28 & 7.20 & 4.04 & 16.55 & 7.68 & 4.20 & 6.03 & 7.29 & 3.08 & 7.69 & 7.10 & 2.13 \\
\hline Ours & 13.63 & 7.14 & 5.10 & 18.62 & 7.72 & 12.57 & 6.88 & 7.34 & 5.13 & 16.44 & 7.52 & 4.77 \\
\hline
\end{tabular}

Note: C, E, T respectively represents Clarity, Entropy and Running time (/s).

\section{Conclusion}

For the purpose of solving such problems as halo and color distortion for the traditional Retinex enhancement algorithm, an improved Retinex image enhancement algorithm based on bilateral filtering was proposed. In the HSI color space, firstly the dynamic range of both original image and illuminant image obtained by bilateral filter was compressed to varying degrees; and then enhanced the reflected image and adjusted saturation S adaptively. Finally, converted the image into RGB 
space. The experimental results indicated that, the proposed algorithm could not only suppress the halo and color distortion, but achieve good effects in detail enhancement and color fidelity. In subsequent work, we will further optimize the algorithm to improve real-time performance, and carry out the research of practical application.

\section{Acknowledgement}

In this paper, the research was sponsored by Natural Science Foundation of Anhui Province (1508085MF110), Scientific and Technological Projects of Anhui Province (1501031102) and Key Basic Research Projects in Colleges and Universities of Jiangsu Province (13KJA510004).

\section{References}

[1] CHEN W. Research of image enhancement algorithm based on Retinex theory [D]. Nanjing University of Science and Technology, 2006: 12-13.

[2] LIU J, ZHAO Y, HU F. A nonlinear image enhancement algorithm based on single scale retinex [J]. Journal of Shanghai Jiaotong University, 2007, 41(5): 685-688.

[3] FANG S, YANG J, CAO Y. et.al. Local multi-scale Retinex algorithm based on guided image filtering [J]. Journal of Image and Graphics, 2012, 17(7): 748-755.

[4] ZHAGN S, ZEGN P, LUO X, et.al. Multi-scale Retinex with color restoration and detail compensation [J]. Journal of Xi'an Jiao tong University, 2012, 46(4): 32-37.

[5] JI Z, CHEN Q, SUN Q. et.al. Single-scale Retinex image enhancement base on bilateral filtering. [J] Journal of Microelectronics \& Computer, 2009, 26(10): 99-102.

[6] HU W, WANG R, FANG S, et.al. Retinex algorithm for image enhancement based on bilateral filtering [J]. Journal of Engineering Graphics, 2010, 02: 104-109.

[7] JI Z, CHEN Q, SUN Q. et.al. Single-scale Retinex image enhancement base on bilateral filtering [J]. Journal of Microelectronics \& Computer, 2009, 26(10): 99-102.

[8] FU X, SUN Y, LIWANG M, et.al. A novel retinex based approach for image enhancement with illumination adjustment[C] //Proceedings of the 2014 IEEE International Conference on Acoustics, Speech and Signal Processing (ICASSP), Piscataway: IEEE, 2014: 1190-1194.

[9] Lee C H, Shih J L, Lien C C, et al. Adaptive multiscale retinex for image contrast enhancement[C]//2013 International Conference on Signal-Image Technology \& Internet-Based Systems IEEE Computer Society, 2013:43-50.

[10]Jobson D J, Rahman Z U, Woodell G A. Properties and performance of a center/surround retinex [J]. IEEE Transactions on Image Processing, 1997, 6(3): 451-62.

[11]LIN K, WU J, XU L. A survey on color image segmentation techniques [J]. Journal of Image and Graphics, 2005, 10(1): 1-10.

[12]Weiss B. Fast median and bilateral filtering [J]. Acm Transactions on Graphics, 2006, 25(3):519-526.

[13]YU X, ZHAGN Y, DU Q, et.al. Local contrast enhancement algorithm based on histogram modification framework [J]. Journal of Computer and Digital Engineering, 2011, 39(3): 5-8.

[14]ZHENG Z, ZHU M. A method to identify sucrose-crystallizing particles based on image-processing [J]. Journal of Computer Engineering and Applications, 2011, 47:190-193.

[15]GUO X. Research of enhancement algorithm for low-illumination image [D]. South China University of Technology, 2013: 16. 